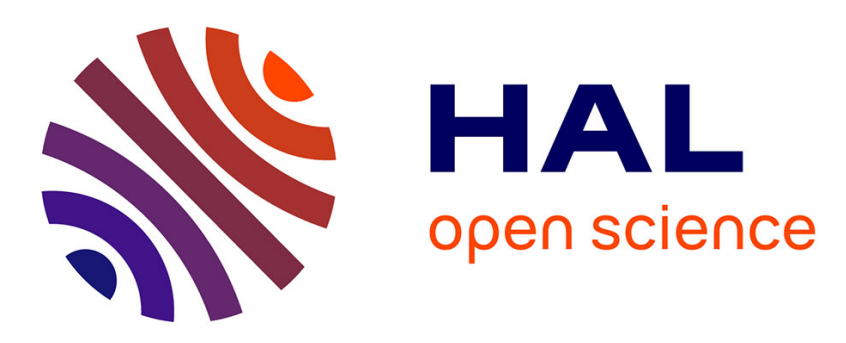

\title{
Weakly nonlinear propagation in thermoacoustic engines: a numerical study of higher harmonics generation up to the appearance of shock waves
}

Come Olivier, Guillaume Penelet, Gaelle Poignand, Joël Gilbert, Pierrick Lotton

\section{To cite this version:}

Come Olivier, Guillaume Penelet, Gaelle Poignand, Joël Gilbert, Pierrick Lotton. Weakly nonlinear propagation in thermoacoustic engines: a numerical study of higher harmonics generation up to the appearance of shock waves. Acta Acustica united with Acustica, 2015, 101 (5), pp.941-949. 10.3813/AAA.918889 . hal-01114410v2

\section{HAL Id: hal-01114410 \\ https://hal.science/hal-01114410v2}

Submitted on 18 Apr 2016

HAL is a multi-disciplinary open access archive for the deposit and dissemination of scientific research documents, whether they are published or not. The documents may come from teaching and research institutions in France or abroad, or from public or private research centers.
L'archive ouverte pluridisciplinaire HAL, est destinée au dépôt et à la diffusion de documents scientifiques de niveau recherche, publiés ou non, émanant des établissements d'enseignement et de recherche français ou étrangers, des laboratoires publics ou privés.

$$
\text { Copyright }
$$




\title{
Weakly nonlinear propagation in thermoacoustic engines: a numerical study of higher harmonics generation up to the appearance of shock waves
}

\author{
C. Olivier, G. Penelet, G. Poignand, J. Gilbert, P. Lotton \\ Laboratoire d'Acoustique de l'Université du Maine, \\ LAUM UMR CNRS-6613, \\ Avenue Olivier Messiaen, 72085 LE MANS cedex9, France.
}

April 21, 2015

\begin{abstract}
Though thermoacoustic engines usually operate at high acoustic amplitude, they rarely exhibit strong deformation of the wavefront due to nonlinear propagation. It has however been demonstrated experimentally that obtaining shock waves in thermoacoustic engines is possible under specific conditions. This paper aims at presenting a simple description of the periodic steady-state operation of thermoacoustic engines describing the wave steepening process leading to shock wave formation. Results of numerical simulations are compared to experimental data in different engine configurations, and model improvements are proposed to reach a realistic description of the weakly nonlinear propagation in thermoacoustic engines.
\end{abstract}

\section{Introduction}

Thermoacoustic engines (TAEs) are thermodynamic systems that make use of a temperature gradient along a porous material to generate self-sustained acoustic oscillations. This auto-oscillation occurs beyond a threshold temperature difference for which the thermoacoustic amplification taking place in the thermoacoustic core compensates exactly all losses in the system. This onset condition is well described by the linear theory of thermoacoustics 11. However, above the onset threshold, the amplitude of the acoustic oscillation grows to reach high levels. The saturation of the growth of amplitude is then controlled by nonlinear phenomena which are the cause for both acoustic and thermal power dissipation. Among the many effects usually described are the acoustically enhanced thermal pumping [1, 2], acoustically induced DC flows [3, 4, 5], complex aerodynamical effects due to geometrical singularities at interfaces between elements of different porosity or at geometric discontinuities [6], or nonlinear propagation leading to higher harmonics generation [7, 8].

Thermoacoustic pumping is well described analytically for simple pore shapes [9], or with hybrid analytic/experimental approaches for more complex materials [10, 11. Acoustic streaming is accounted for in models by higher-order developments of the basic equations [12, 13, and experimental solutions are implemented to limit its effects [14, 15]. The aerodynamical effects at geometrical singularities have been extensively studied numerically [16] and experimentally [17, 18, in thermoacoustic systems, but are often analytically disregarded because of the complexity of the involved phenomena. Some of these effects may be minimized empirically by the use of artifacts such as flow straighteners [14].

Nonlinear propagation due to the high acoustic amplitudes can be dealt with by a proper design of resonators [19. It is usually ignored in the description of thermoacoustic engines for several reasons. Firstly, when present, the magnitude of the losses due to nonlinear propagation is small compared to the one produced by other nonlinear phenomena. Also, most engines have complex geometries making their resonances inharmonic [20]. Even when built with a simpler shape of resonator such as a straight tube, the presence of the steep temperature gradient tends to make the frequencies of the higher modes non-integer multiples of the fundamental resonant frequency $f_{0}$ of the engine. Therefore, the formation of shock waves or a strong deformation of the wavefront is rarely observed in thermoacoustic engines, although the acoustic pressure oscillations may reach an amplitude of $10 \%$ of the static pressure [21, 22]. Nevertheless, it has been reported by Biwa et al. 23. that is it possible to obtain an experimental traveling shock wave in an annular thermoacoustic engine under specific conditions. Furthermore, the same team showed more recently that shock wave formation is also possible in a standing wave engine 24.

This paper proposes a numerical investigation of the nonlinear behavior of simple standing or traveling wave thermoacoustic engines such as the ones presented schematically in Fig. 1. In Section 2, the analytic tools used in this study are briefly presented, for the description of the propagation in the thermoacoustic core with the linear equations of thermoacoustics [1] and for the nonlinear propagation in the waveguides with generalized Burgers equation in the form introduced by Sugimoto 25 . A numerical solving process of these equations of propagation is proposed, based on the one used by Menguy and Gilbert 26] for the case of forced oscillations and adapted 
(A)
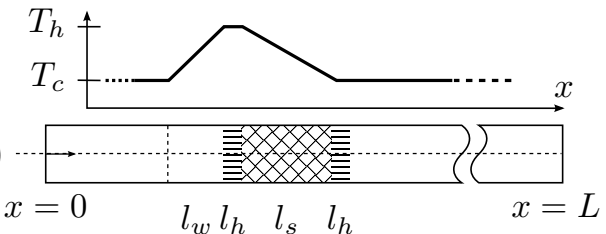

(B)

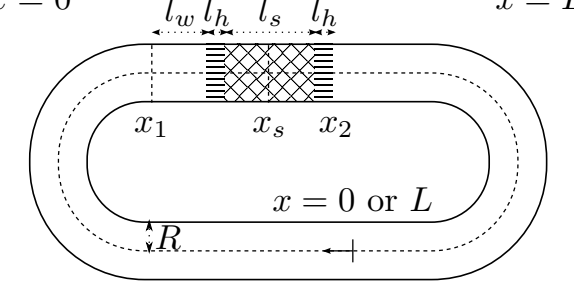

Figure 1: Architectures of the thermoacoustic engines under consideration in this study: (A) Standing-wave engine made of a thermoacoustic core (TAC) in a resonant waveguide; (B) Traveling-wave engine made of a similar TAC in a looped tube. On top is the temperature distribution imposed in both engines, assuming linear variations. The TAC consists of two heat exchangers (striped), a stack (hatched) and the part of the resonator comprising a temperature gradient, referred to as the thermal buffer tube (TBT).

to the case of self-sustained oscillations of undetermined frequency. Section 3 is devoted to presenting numerical results for different engine configurations. We show that higher harmonics generation can be favored or inhibited for various parameters of the thermoacoustic core (geometry, temperature distribution $T_{0}(x)$, position in the resonator $x_{s} / L$, etc.). Computed steady-state waveforms are presented that show the possibility of shock wave formation for both standing and traveling wave configurations under particular conditions. These results are discussed in relation with available experimental results 23, 24. A brief discussion is also given to interpret these results. The conclusion presented in Section 4 highlights the main results and proposes perspectives to this study.

\section{Simplified approach for the de- scription of TAEs}

A basic treatment of the nonlinear propagation in thermoacoustic engines (TAEs) suggests a separation in two sub-problems: on one hand, the simplified description of the nonlinear propagation in the temperaturehomogeneous waveguide and, on the other hand, the description of the propagation in the idealized thermoacoustic core comprising an inhomogeneous temperature distribution. Analytic tools are available for both problems: nonlinear propagation in waveguides is expressed making use of generalized Burgers' equations [27, 28, and the propagation in the thermoacoustic core (TAC) is given by Rott's linear theory of thermoacoustics [1]. The coupling of the subsystems and the research of a solution with an iterative harmonic balance method allows to compute the amplitudes for all harmonics of the periodic steadystate regime, for a given set of geometrical parameters, thermophysical properties and an assigned temperature distribution through the thermoacoustic core.

\subsection{Nonlinear propagation in waveguides}

The nonlinear propagation of waves in ducts has since long been described, with application to various fields 29, 30, 31. In the context of this study, the work of Menguy and Gilbert 26 is worth considering. Their use of the formalism of generalized Burgers' equations to describe the lossy, weakly nonlinear acoustic propagation allowed them to emphasize the shock wave formation in wind instruments. This phenomenon, previously observed experimentally by Hirschberg et al. 32 is now known to explain the brassiness of their tone 33 . The same approach is used in this study to describe the cascade process of higher harmonics generation, applied to the thermoacoustic auto-oscillation whose frequency is likely to drift with the variations of amplitude of the acoustic variables. In a fluid characterized by its shear dynamic and kinematic viscosities $\mu$ and $\nu$, its bulk viscosity $\eta$, its thermal conductivity $\kappa$ and its specific heat capacity $c_{P}$ (all five parameters being assumed constant in absence of a temperature gradient), the acoustic plane wave of angular frequency $\omega$ propagating in a resonator of radius $R$ at the typical value $c_{0}$ of the sound speed in that fluid may be characterized by three numbers:

- $M=u_{0} / c_{0}$, the acoustic Mach number, which is the ratio of the peak amplitude of the particle velocity to the speed of sound;

- Re $=u_{0} / \sqrt{\nu \omega}$, an acoustic Reynolds number 34, which is the ratio of inertia to viscous forces and qualifies the type of flow in the boundary layer thickness $\delta_{\nu}=\sqrt{\nu / \omega}$

- $S h=\sqrt{\nu / \omega} / R$, the shear number, which is the ratio of the viscous boundary layer thickness to the characteristic transverse dimension of the waveguide.

Thermoacoustic engines usually operate at frequencies from tens to a few hundreds of hertz, with waveguides of a few centimeters in radius, and high sound levels (145$175 \mathrm{~dB}_{\text {SPL }}$ with a working fluid at atmospheric pressure, that is drive ratios - ratio of acoustic pressure amplitude $|p|$ to the static pressure $P_{0}$ - from $0.5 \%$ up to $10 \%$ ). Under these conditions, the three dimensionless numbers $M$, $1 / R e$ and $S h$ are small compared to unity, indicating that the nonlinear effects of propagation are not local but cumulative [26], with two consequences. Firstly, any acoustic wave may be considered as the superposition of two noninteracting counterpropagating waves, whose propagation is described by two independent equations. Secondly, this allows to separate two scales of time and space for the description of the propagation, one fast scale (resp. short) for the quasi-linear local acoustic propagation, and one slow scale (resp. long) for the cumulative effects. Furthermore, the comparison of the relative amplitudes of the dimensionless numbers allows to evaluate the importance of the different phenomena taking place during propagation. A diagram of predominance is given in Fig. 2, allowing to visualize the domain of validity of the chosen 


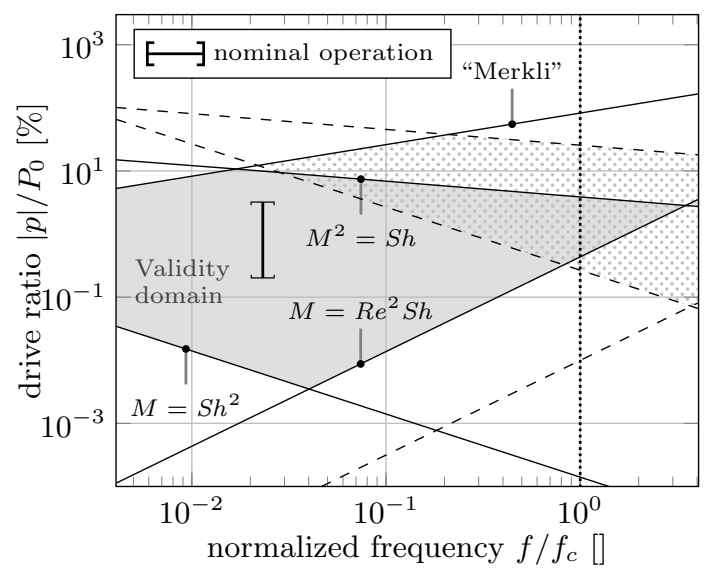

Figure 2: Predominance diagram in the amplitude vs. frequency plane for a cylindrical tube of $4 \mathrm{~cm}$ in diameter filled with air at ambient pressure. Each straight line represents the limit of predominance of one phenomenon over another: Merkli condition is the upper limit for laminar flow in boundary layers; above $M^{2}=S h$, second order effects take over boundary layer effects; $M=S h^{2}$ and $M=R e^{2} S h$ are two limits under which a nonlinear description of the propagation is not necessary. Parallel dashed lines represent the same conditions in a pore of the stack. Adapted from Fig. 1 of [26].

approach as a function of frequency and drive ratio. Conditions $M=S h^{2}$ and $M=R e^{2} S h$ represent the limits under which nonlinear phenomena are small compared to the neglected linear effects (e.g. the bulk losses or the effects of the curvature of the waveguide), and a linear description of acoustic propagation would suffice. Condition $M^{2}=S h$ stands as the limit above which boundary layer effects are to be ignored compared to second order effects, better described by another approach than the one used here. Merkli condition $R e=u_{0} / \sqrt{\nu \omega}<400$ is the limit under which the flow in the boundary layers is laminar, a necessary condition for the expression of the boundary layer losses used thereafter. The cut-off frequency of the waveguide for plane waves is given as the vertical dotted line for information. The description given here is that of a one-dimensional system (only plane waves propagate and the amplification from the thermoacoustic core is 1D) and therefore does not account for transverse modes, though the frequency of the higher harmonics considered can be above that cut-off frequency.

These limits border a domain, represented by the shaded area in Fig. 2, which comprises the condition of nominal operation of the two studied thermoacoustic engines. The acoustic propagation in the resonators may therefore be described by the two independent generalized Burgers' equations 25.

$$
\partial_{\sigma} p_{ \pm} \mp p_{ \pm} \partial_{\theta_{ \pm}} p_{ \pm}= \pm \frac{S t}{\epsilon} \partial_{\theta_{ \pm}}^{2} p_{ \pm} \mp \frac{T}{\epsilon} \partial_{\theta_{ \pm}}^{\frac{1}{2}} p_{ \pm}
$$

where $\sigma$ is a long spatial scale and $\theta$ a delayed time scale 35. Subscript + (resp. - ) stands for propagation in the positive (resp. negative ) $x$ direction. $\epsilon=M \frac{\gamma+1}{2}$ is an evaluation of the importance of the nonlinear effects. Stokes' number $S t=\frac{M^{2}}{2 R e^{2}}\left(\frac{4}{3}+\frac{\eta}{\nu} \frac{\gamma-1}{P r}\right)$, where $\operatorname{Pr}=\frac{\mu c_{P}}{\kappa}$, stands for the importance of bulk losses, that are proportional to the square of the frequency (as is emphasized by the double derivative in the time domain). This last term is negligible for small deformations of the wavefront, but cannot be ignored any more once the shock is formed. The term with the fractional derivative stands for the thermoviscous losses taking place in the boundary layer, whose relative importance is designated by $T=S h\left(1+\frac{\gamma-1}{\sqrt{P r}}\right)$ and proportional to the square root of the frequency. The $\frac{1}{2}$ fractional derivative is defined as the convolution product

$$
\begin{aligned}
\partial_{\theta}^{\frac{1}{2}} p & =\partial_{\theta} p * \frac{1}{\sqrt{\pi \theta}} \\
& =\frac{1}{\sqrt{\pi}} \int_{-\infty}^{\theta} \frac{1}{\sqrt{\theta-\theta^{\prime}}} \partial_{\theta^{\prime}} p\left(\theta^{\prime}, \sigma\right) \mathrm{d} \theta^{\prime} .
\end{aligned}
$$

\subsection{Propagation through the TAC}

By thermoacoustic core (TAC), we designate the parts of the engine between $x=x_{1}$ and $x=x_{2}$ (see Fig. 1) comprising an inhomogeneous temperature distribution and consisting of an ambient heat exchanger (AHX), a porous medium designated as the stack, submitted to a temperature gradient by a hot heat exchanger (HHX) and a thermal buffer tube (TBT) which provides a thermal buffer between the HHX and the ambient temperature waveguide. On the diagram of predominance (Fig. 2), the limits of predominance are also represented in dashed lines for the propagation of an acoustic wave in a pore of the stack (or of one of the heat exchangers, of equivalent hydraulic radius). The nominal working conditions of the engine are below the validity domain of the approach presented above, represented by the dotted area. More particularly, the working conditions are below the $M=S h^{2}$ condition, indicating that a nonlinear description of the propagation in the stack is not necessary as long as the predominant boundary layer effects are well accounted for. Moreover, in the engine core, the presence of a strong temperature gradient $T_{0}(x)$ does not allow the decoupling of the acoustic propagation into two counterpropagating waves and the temperature dependence of the thermoviscous properties of the fluid must be accounted for. Therefore, the TAC will be described with a different approach than the rest of the engine. In the frequency domain, it can be described by a scattering matrix 36 obtained from the classical equations of thermoacoustics [1, and expressing the counterpropagating components for each harmonic $p_{ \pm}^{(n)}$ of frequency $n f_{0},(n \in \llbracket 1, \infty \llbracket)$ of the acoustic pressure variations at one extremity of the core $\left(x=x_{2}\right)$ as a function of the same variables at the other end $\left(x=x_{1}\right)$, with help of the reflection and transmission coefficients $\mathcal{R}^{ \pm}$and $\mathcal{T}^{ \pm}$ of the core

$$
\begin{aligned}
\left(\begin{array}{l}
\tilde{p}_{+}^{(n)}\left(x_{2}\right) \\
\tilde{p}_{-}^{(n)}\left(x_{1}\right)
\end{array}\right) & =\mathcal{S}\left(n f_{0}, T_{0}(x)\right)\left(\begin{array}{l}
\tilde{p}_{+}^{(n)}\left(x_{1}\right) \\
\tilde{p}_{-}^{(n)}\left(x_{2}\right)
\end{array}\right) \\
& =\left[\begin{array}{ll}
\mathcal{T}^{+} & \mathcal{R}^{-} \\
\mathcal{R}^{+} & \mathcal{T}^{-}
\end{array}\right]\left(\begin{array}{l}
\tilde{p}_{+}^{(n)}\left(x_{1}\right) \\
\tilde{p}_{-}^{(n)}\left(x_{2}\right)
\end{array}\right)
\end{aligned}
$$

Coupling this description of the acoustic propagation through the thermoacoustic core together with the ex- 
pression of the nonlinear propagation in the temperature homogeneous waveguides is sufficient to obtain the saturated waveforms of a thermoacoustic engine. However, as stated in Section 1, various phenomena of heat and mass transfer work together to dissipate energy in TAEs. Ignoring the additional losses generated by these phenomena would yield numerical amplitudes obtained with the current linear description of the thermoacoustic core beyond the observed experimental saturation levels of the acoustic amplitudes. It is therefore necessary to include some nonlinear effects in the description of the TAC for the solution to converge to realistic amplitudes of saturation. Among the previously cited effects, sudden changes of the effective cross-section area at porous elements interfaces are the source of localized dissipation of acoustic energy that results in a pressure drop $\Delta p$ through the TAC. These so-called minor losses may be evaluated roughly by extrapolating to oscillatory flows [37] the DC behavior of such interfaces, yielding an average pressure drop for each harmonic

$$
\Delta p^{(n)}(t) \simeq-\frac{4}{3 \pi} \frac{\rho_{0}}{2}\left(K_{12} \phi_{1}+K_{21} \phi_{2}\right)\left|u^{(n)}\right|^{2}(t),
$$

where $\rho_{0}$ is the density of the fluid at the temperature of the interface, $\phi_{1}$ and $\phi_{2}$ the porosity of the elements on each side of an interface, and $K_{12}$ and $K_{21}$ are tabulated losses coefficients for a sudden section expansion or contraction 38. The acoustic velocity $u$ is evaluated from Euler's equation: $u(t) \simeq\left(p_{+}(t)-p_{-}(t)\right) /\left(\rho_{0} c_{0}\right)$. Taking into account those losses proportional to $u^{2}$ yields the nonlinear scattering matrix for the thermoacoustic core

$$
\left(\begin{array}{l}
\tilde{p}_{+}^{(n)}\left(x_{2}\right) \\
\tilde{p}_{-}^{(n)}\left(x_{1}\right)
\end{array}\right)=\mathcal{S}_{n l}\left(n f_{0}, T_{0},\left|\tilde{u}^{(n)}\right|^{2}\right)\left(\begin{array}{l}
\tilde{p}_{+}^{(n)}\left(x_{1}\right) \\
\tilde{p}_{-}^{(n)}\left(x_{2}\right)
\end{array}\right) .
$$

One should note that such a description is not intended to be accurate, but should give an order of magnitude of the encountered losses in the TAC, allowing realistic levels of saturation. Neglecting the other nonlinear saturating effects is a choice to keep the description simple. It can however be justified by the following reasoning: aerodynamical edge effects, acoustic streaming and thermoacoustic pumping reduce the efficiency of thermal-to-acoustic conversion by causing heat transfer via convection and/or conduction. Thus, their effect would merely translate into a variation of the temperature of the hot heat exchanger. However, the description adopted in the frame of this paper concentrates on steady-state regime and therefore the imposed temperature of the hot heat exchanger can be considered as the resulting one, accounting for these effects.

\subsection{Solving process}

Though approximate solutions are available 39, equations (11) describing the nonlinear propagation of quasiplane waves in a thermoviscous fluid do not have any exact analytic solution. They are solved numerically in the frequency domain for the different sections of waveguide $\left[0 ; x_{1}\right]$ and $\left[x_{2} ; L\right]$, coupled by the scattering matrix (5), and with boundary conditions depending on the geometry of the engine. The solution is sought in the form of a harmonic series

$$
\tilde{p}=\sum_{n=1}^{+\infty}\left[a_{n}(\sigma) \sin n \theta+b_{n}(\sigma) \cos n \theta\right],
$$

the introduction of which in the lossy Burgers' equation (11) and term-by-term equalization yields a set of equations for the coefficients $a_{n}$ and $b_{n}$. This set is solved numerically following the steps stated here after, which consist in an extension to self-sustained oscillations of the work of Menguy and Gilbert [26] for forced oscillations.

- (i) The initial harmonic spectrum is defined with a fundamental frequency close to the resonant frequency of the engine (e.g., at the resonance frequency of the empty resonator) with an amplitude of the fundamental of a few hundreds of $\mathrm{Pa}$ and 0 for all harmonics, at one extremity of the engine.

- (ii) Each harmonic of the signal is propagated through the whole engine back and forth to the starting point by applying a backwards finite differences scheme on equations (11) in the resonator parts, and thanks to the scattering matrix (5) through the TAC.

- (iii) After one complete cycle, the amplitudes of each harmonic of the final spectrum are compared elementwise to the amplitudes of the initial spectrum. In the same manner, the phase of the fundamental component is compared between the initial $\phi_{i}$ and final states $\phi_{f}$.

- (iv) If the phase difference between initial and final states of the fundamental is not zero (i.e, if $\phi_{f}-\phi_{i} \neq 0$ $\bmod [2 n \pi]$, with $n$ depending on the working mode), the frequency of the fundamental is adjusted. Due to the nonlinear drift of the resonant frequency with the amplitude of the acoustic pressure (sometimes called nonlinear detuning [8]), this correction has to be repeated at each iteration of the solving process, contrarily to the case of forced oscillations where the frequency is imposed by a driving source.

- (v) If the difference $e$ of amplitudes (in the sense of the absolute deviations) between initial and final states is smaller than a predefined small threshold $\varepsilon$, the obtained solution is considered to be the steady-state saturated spectrum and the search of a solution stops here. If $e>\varepsilon$, the steady-state has not been reached yet. The spectrum obtained after this iteration is used to initiate the next iteration and steps (ii) through (v) are repeated until a satisfying solution is found (see Ref. [26] for a more detailed description of the procedure in the case of forced oscillations).

The definition of the system is such here that it tends to the point where losses compensate the thermoacoustic amplification for all harmonics after a sufficient number of iterations, whatever the initial state provided. In practice, in order to reduce the computation time, the number of computed harmonics has to be limited to a reasonable 


\begin{tabular}{|c|c|c|c|c|c|}
\hline \multicolumn{3}{|c|}{ Resonator } & \multicolumn{3}{|c|}{ Stack } \\
\hline \multirow[t]{2}{*}{$L$} & A & $3.04 \mathrm{~m}$ & $l_{s}$ & & $2.0 \mathrm{~cm}$ \\
\hline & B & $0.84 \mathrm{~m}$ & $y_{0 s}$ & A & $0.47 \mathrm{~mm}$ \\
\hline \multirow[t]{2}{*}{$x_{s} / L$} & A1 & 0.14 & & B & $0.75 \mathrm{~mm}$ \\
\hline & A2 & 0.22 & $\phi$ & A & 0.88 \\
\hline \multicolumn{3}{|c|}{$R \quad 2.05 \mathrm{~mm}$} & & B & 0.74 \\
\hline \multicolumn{3}{|c|}{ Heat exchangers } & pore & & square \\
\hline \multicolumn{2}{|c|}{$l_{h}$} & $1.0 \mathrm{~cm}$ & \multicolumn{3}{|c|}{ TBT } \\
\hline \multirow{2}{*}{\multicolumn{2}{|c|}{$y_{0 h}$}} & $0.5 \mathrm{~mm}$ & $l_{w}$ & A & $30 \mathrm{~cm}$ \\
\hline & & 0.66 & & B & $3.2 \mathrm{~cm}$ \\
\hline \multicolumn{2}{|c|}{$\begin{array}{l}\varphi_{h} \\
\text { pores }\end{array}$} & slits & $P_{0}$ & air & $100 \mathrm{hPa}$ \\
\hline
\end{tabular}

Table 1: Engines parameters for numerical simulations. A1 and A2 are two configurations of the same standing wave engine A 24]; B is a traveling wave engine [23]. Both engines are based on similar parts except for the stack geometry (different porosity $\phi$, and hydraulic radius $y_{0}$ ) and work with air at ambient pressure.

number. However, since the energy content of each harmonic decreases with its rank due to volumetric losses proportional to $\omega^{2}$, the truncation error drops quickly when the number of harmonics is increased over a few tens.

\section{Results and discussion}

Two classical engines are modeled for this study. The configurations are chosen to describe actual engines for which experimental results showing shock wave formation are available 23,24 . The first one $(\mathrm{A})$ is a standing wave engine closed at both ends [24]. The second one (B) is a quasi-traveling wave engine with a looped resonator, using a core of similar construction, but with a stack with slightly different specifications [23]. The dimensions of both engines are provided in Table 11. The length $l_{w}$ of the TBT is not defined in either reference, because no secondary heat exchanger is used to constrain the cold temperature in the resonators. In the quasi-traveling wave engine (B), the amplification of the TAC can be highly sensitive to the temperature distribution [36. Assuming a linear temperature distribution $T_{0}(x), l_{w}$ is then defined to match the given experimental onset threshold of the experimental apparatus. In the standing wave engine however, the sensitivity to the temperature distribution appears to be much less important. The TBT is chosen to be short enough to allow the placement of the core close to the extremity of the engine.

It is to be noted that, though the data provided in Ref. [23, 24] is admittedly sufficient for the description of the linear behavior of the engines (i.e., their onset threshold), it is not enough for a precise rendering of their operation above threshold. Some parameters are not provided in the description of the experimental configurations, either because they were not relevant in the frame of the presented work or because they were not available to the authors (e.g., the temperature distribution in the whole TAC); these parameters are nonetheless important to describe theoretically the steady-state operation above threshold. These above-mentioned experimental configurations are however used as reference for this study, still allowing for a qualitative comparison. The description of the nonlinear effects controlling the amplitude of saturation in the model presented in this paper provides only an estimate of their amplitude, and therefore does not allow for a quantitative comparison.

The computed periodic steady state waveform in a thermoacoustic engine described with the previous tools is the result of the balance of the phenomena taking place in the two sections of the engine. On one hand, the nonlinear propagation of high amplitude acoustic waves induces the cascade of energy from the fundamental frequency of the periodic oscillation regime to its higher harmonics. This phenomenon prevails in the temperature homogeneous resonant parts of the engine. With the generation of higher harmonics also comes additional thermal and viscous dissipation, increasing with frequency. These losses are the main source of saturation of the amplitude, together with the minor losses in the TAC. On the other hand, from the linear theory of thermoacoustics, a thermoacoustic core with a given set of parameters (geometry, temperature distribution $T_{0}(x)$, position in the resonator $x_{s} / L$, etc.) can be seen either as a source or a sink of volume flow rate 6. Its amplification gain (resp. positive or negative) is dependent on the acoustic impedance within the stack 40, and therefore is different for each harmonic of a wave. Indeed, the acoustic impedance is dictated by geometrical considerations, including the boundary conditions at the extremities of the engine.

To favor the wavefront deformation and possibly lead to the formation of a shock wave, balance has to be achieved for each harmonic between the possible amplification from the core and the increase of energy caused by the cascade from the lower harmonics on one hand, and the losses, the decrease of energy due to the cascade to higher harmonics and the possible attenuation from the core on the other hand.

\subsection{Quasi-standing wave engine}

Figure 3 shows the progressive deformation of the steadystate wavefront with the increase of the imposed temperature difference $\Delta T=T_{h}-T_{h, o}$ above the onset threshold for two different positions of the core in the resonator of the standing wave engine (configuration (A) of Fig 1). In configuration (A1) the core is placed close to the extremity $\left(x_{s} / L=0.14\right)$ (which corresponds to the final experimental configuration of Ref. 24]), whereas in configuration (A2) the core is closer to the center $\left(x_{s} / L=0.22\right)$. At first glance, one can see that in both cases the wavefront distorts progressively as the imposed temperature difference increases. However for an amplitude of the pressure oscillations corresponding to a drive ratio (that is ratio of the peak-to-peak amplitude of pressure oscillations to the static pressure) of $10 \%$, configuration (A1) clearly exhibits formed shocks - the maximum slope shown by the vertical dashed line tends to infinity - when configuration (A2) does not - the maximum slope is finite -, as one can see for the cases in bold black on Figure 3 . Configuration (A1) appears more suitable for shock formation. 

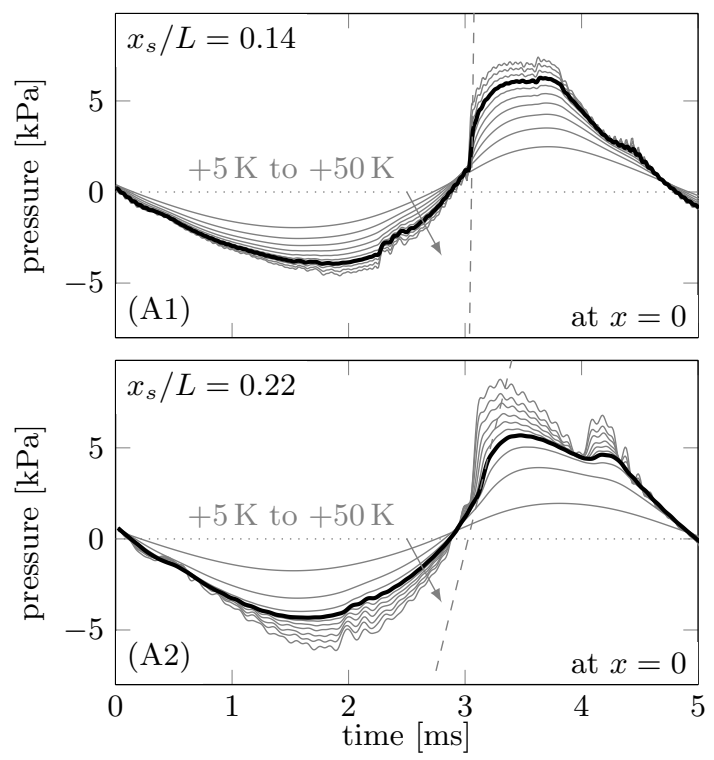

Figure 3: Computed deformation of the wavefront for two different positions $x_{s} / L$ of the core in the resonator of the standing wave engine (configuration (A) of Fig. 1) for increasing temperature differences beyond threshold from $5 \mathrm{~K}$ to $50 \mathrm{~K}$ with a step of $5 \mathrm{~K}$. (A1): $x_{s} / L=0.14$, (A2): $x_{s} / L=0.22$. The case highlighted in black is for the same drive ratio of $10 \%$. Dashed lines stand for maximum slope in each case, showing that a shock is formed in the upper case (infinite slope), and not in the lower one (finite slope).

Figure 4(a) shows the saturated waveforms for higher temperature differences in the configuration (A1) where the shock forms more easily (i.e. when the thermoacoustic core is closer to the extremity, $x_{s} / l=0.14$ ). Once the shock is formed, its amplitude continues to increase. The peak amplitude however grows more slowly as more energy is dissipated by the shock for higher $\Delta T$. At high amplitudes, one should note the presence of irregularities in the waveform. The large sharp accidents (circled on Fig. 4(a)) are not numerical artifacts. They remain present no matter how many harmonics are taken into account in the computation and are independent of the spatial discretization of the problem; they are more likely due to the multiple reflections caused by the presence of the steep temperature gradient and the porous elements interfaces in the TAC. One can also distinguish small "high frequency" oscillations (emphasized by the zoomed portion on Fig. 4.(a)). They are the result of the harmonic series truncation; the energy carried by the truncated part of the spectrum is rejected on the last computed harmonics. Figure 4.(b) illustrates the temporal evolution of the saturated shock wave at regularly spaced points in the engine. The spatio-temporal evolution of the shock in the quasistanding wave engine is the result of the two counterpropagating waves of equivalent amplitudes propagating back and forth, being reflected on its rigid terminations. The propagation of the shock in the engine is highlighted by the arrows. One acoustic period last for one round trip of the shock in the engine, showing that the engine works on its first mode - the half-wavelength mode. This results
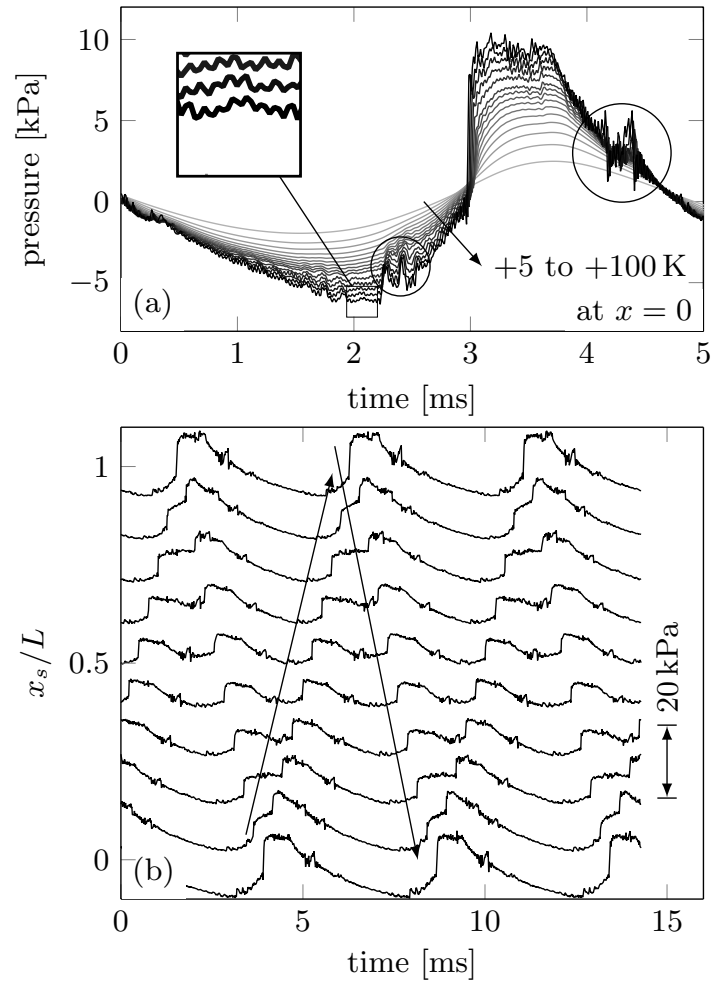

Figure 4: (a): Acoustic pressure waveform computed in the standing wave engine $(\mathrm{A} 1)$ when the TAC is placed at position $x_{s} / L=0.14$, for increasing temperature differences beyond threshold from 5 to $100 \mathrm{~K}$ with a step of $5 \mathrm{~K}$ up to $50 \mathrm{~K}$, then $10 \mathrm{~K}$, shown at position $x=0$.

(b): Spatio-temporal evolution of a quasi-standing shock wave computed for $\Delta T=100 \mathrm{~K}$ in the engine configuration (A1). One can clearly see a shock wave going back and forth in the engine as emphasized by the arrows. 

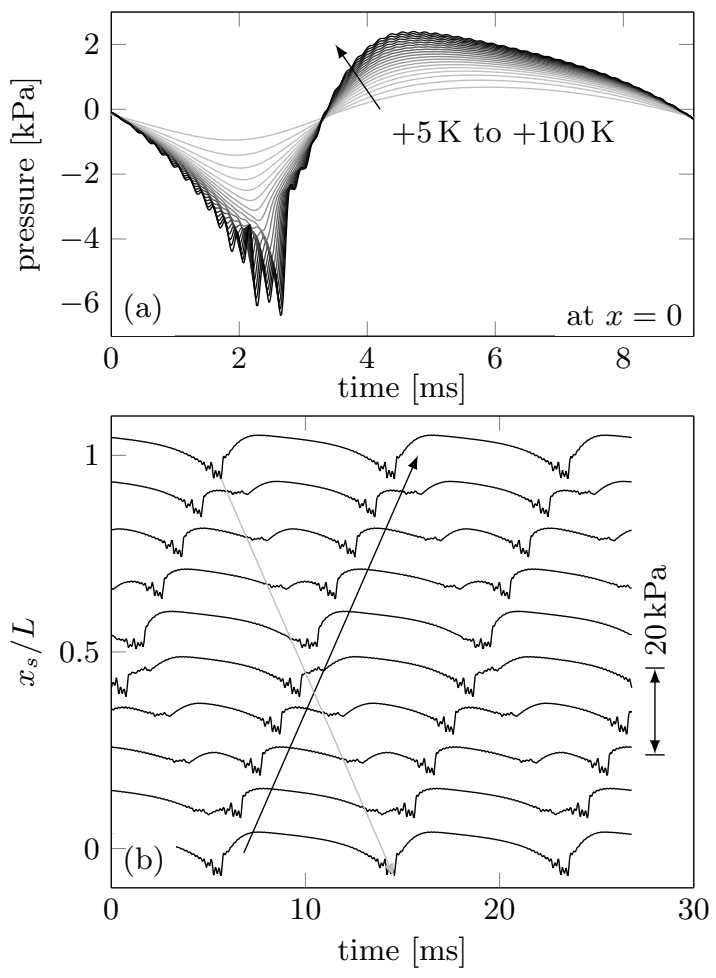

Figure 5: (a): Acoustic pressure waveform computed in the quasi-standing wave engine (B) for increasing temperature differences from 5 to $100 \mathrm{~K}$ beyond threshold at position $x=0$.

(b): Spatio-temporal evolution of a quasi-traveling shock wave computed for $\Delta T=\Delta T_{o}+100 \mathrm{~K}$ in the engine configuration (B) of Fig. 1. One can clearly see a shock wave traveling clockwise in the engine (black arrow) as represented in Fig. 1. One can also distinguish a wavefront propagating counterclockwise (gray arrow).

are to be compared with those obtained experimentally by Biwa et al. [24. The computed waveforms present a similar temporal evolution, and amplitude of the same order of magnitude to the measured ones; the model seems to have captured the nonlinear behavior of the wave propagation.

\subsection{Quasi-traveling wave engine}

Figure 5 shows in the same fashion the numerical results for the configuration of quasi-traveling wave engine (B). In this case however, it is worth mentioning that the engine is spatially translation invariant; that is, the position of the core is not relevant to describe the behavior of the engine. Fig. 5. (a) shows the progressive formation and growth of the shock with the increase of the imposed temperature difference above threshold $\Delta T$. Fig. 5. (b) shows a formed traveling shock wave going around the looped engine as emphasized by the black arrow, operating one rotation around the engine per period because the engine is working on its first mode - the one-wavelength mode. Signals at positions 0 and 1 are identical. Since the wave is not purely traveling [41, a small counterpropagating wavefront can also be observed, emphasized by the gray line. These results are to be compared with those obtained experimentally by Biwa et al. for a similar configuration
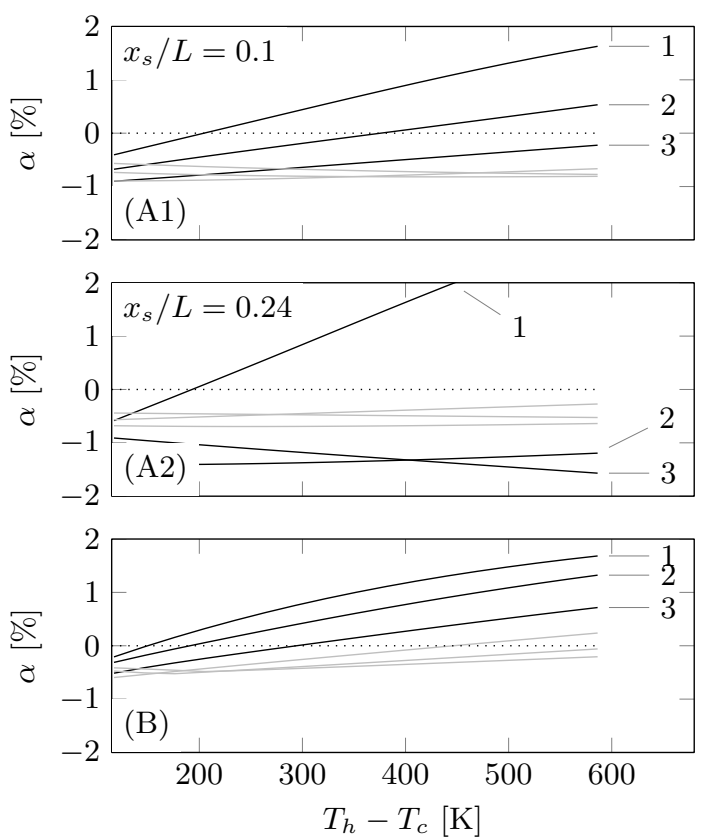

Figure 6: Amplification rate $\alpha$ of the three first modes of the engines versus the temperature gradient imposed for the three configurations (A1), (A2), and (B). Modes 4 to 6 are also given in gray. A positive amplification rate means that a wave at the frequency of the corresponding mode will be amplified by the TAC. $\alpha=0$ for mode 1 represents the onset threshold, for which $T_{h}-T_{c}=\Delta T_{o}$.

[23, where a good qualitative agreement is observed.

\subsection{A linear interpretation}

To attempt an interpretation of the results presented above, one can rely - at least partially and cautiously on a linear analysis of the amplification of the thermoacoustic engines. To this end, we will rely on the method presented in Ref. 42]: the amplification rate $\alpha$ is given by the imaginary part of the complex frequency obtained from the linear transfer matrix of the thermoacoustic engine. Figure 6] shows the amplification rate $\alpha$ for the first modes of the three different configurations of engines. If $\alpha>0$, the mode is amplified by the thermoacoustic core; that is the core acts as a source of energy at that frequency. If $\alpha<0$, the thermoacoustic core behaves as an energy sink and the wave is attenuated. Though the frequencies of the higher modes do not correspond strictly to those of the harmonics of the working mode - mainly because of inharmonicity induced by the presence of the temperature gradient -, they are still close enough for a qualitative analysis of the behavior of the harmonics in the engine.

One can notice that the first modes are more amplified with the increase of the imposed temperature difference along the stack in both cases (A1) and (B), corresponding respectively to the standing wave engine with the core close to the extremity and the traveling wave engine. This means that the greater the temperature difference is, the less the energy cascaded from the fundamental frequency will be attenuated in the TAC, thus facilitating the wave- 
front deformation. Case (A2) however shows a different behavior: though the fundamental frequency of the working mode seems more easily amplified in this configuration, the second and third harmonics are always (linearly) attenuated. The energy cascaded from the fundamental frequency will then be dissipated in the thermoacoustic core, limiting its transit to the superior harmonics and consequently prevent the deformation of the wavefront. Finally, the shock wave generation in a thermoacoustic engine appears to be related to the attenuation or amplification in its core of the higher harmonics produced by the energy cascade from the fundamental frequency. In cases (A1) and $(\mathrm{B})$, the thermoacoustic core acts as an amplifier for the higher harmonics, supporting the wavefront deformation, whereas in case (A2), it behaves as an energy sink, creating a barrier for energy cascade impeding the deformation. Because such an interpretation is based on observing the variations of an amplification rate defined in the frame of a linear theory, it has to be considered with caution. Nevertheless, this interpretation is also the one supported by Biwa et al. 23, 24, from the measurements of intensity at both side of the TAC. This work furthermore brings a numerical approach that successfully renders the nonlinear behavior of the studied engines, as well as an a priori reading of the linear model of the acoustic propagation to support this interpretation.

\section{Conclusion}

In this paper, a numerical simulation of the nonlinear propagation in thermoacoustic engines of basic geometry is presented. It uses the formalism of generalized Burgers' equations for nonlinear propagation in the temperature homogeneous waveguide sections and the linearized equations of thermoacoustics in the engine's core, solved numerically to obtain the periodic steady-state regime of auto-oscillation. This expressions proved to be sufficient for a qualitative description of the wave steepening in thermoacoustic engines of simple geometry, for both standing and quasi-traveling wave configurations. Numerical results show good qualitative agreement with experimental results obtained in 23, 24.

Furthermore, a more accurate description of the thermoacoustic core should help to obtain a better quantitative match. Notably, using a nonlinear profile temperature, both in the stack and the TBT, will influence the amplification rate of the core, particularly in the quasitraveling wave engine. Besides, the description of minor losses is quite rough in this work, only aiming at getting an order of magnitude of the losses. A more accurate description is important for the prediction of the levels of saturation, and should be accompanied by more exhaustive evaluation of the thermophysical parameters in the experimental configuration, such as the presence of acoustically induced streaming or the spatial distribution of temperature in the different elements composing the core. A quantitative comparison would be possible only under these conditions.

\section{Acknowledgments}

The authors wish to acknowledge A. Hirschberg for his help and precious advice in the redaction of this paper.

\section{References}

[1] N. Rott, "Thermoacoustics," Adv. Appl. Mech., vol. 20, pp. 135-175, 1980.

[2] T. Yazaki, A. Tominaga, and Y. Narahara, "Large heat transport due to spontaneous gas oscillation induced in a tube with steep temperature gradients," J. Heat Transfer, vol. 105, no. 4, pp. 889-894, 1983.

[3] C. Desjouy, G. Penelet, P. Lotton, and J. Blondeau, "Measurement of acoustic streaming in a closed-loop traveling wave resonator using laser doppler velocimetry," J. Acoust. Soc. Am., vol. 126, p. 2176, 2009.

[4] M. W. Thompson and A. A. Atchley, "Simultaneous measurement of acoustic and streaming velocities in a standing wave using laser doppler anemometry," J. Acoust. Soc. Am., vol. 117, p. $1828,2005$.

[5] S. Moreau, H. Bailliet, and J.-C. Valière, "Measurements of inner and outer streaming vortices in a standing waveguide using laser doppler velocimetry," J. Acoust. Soc. Am., vol. 123, no. 2, pp. 640-647, 2008.

[6] G. W. Swift, Thermoacoustics: a unifying perspective for some engines and refrigerators. Acoustical Society of America, 2002.

[7] H. Yuan, S. Karpov, and A. Prosperetti, "A simplified model for linear and nonlinear processes in thermoacoustic prime movers. part ii. nonlinear oscillations," J. Acoust. Soc. Am., vol. 102, p. 3497, 1997.

[8] V. Gusev, H. Bailliet, P. Lotton, and M. Bruneau, "Asymptotic theory of nonlinear acoustic waves in a thermoacoustic primemover," Acta Acust. united Ac., vol. 86, no. 1, pp. 25-38, 2000.

[9] W. P. Arnott, H. E. Bass, and R. Raspet, "General formulation of thermoacoustics for stacks having arbitrarily shaped pore cross sections," J. Acoust. Soc. Am., vol. 90, p. 3228, 1991.

[10] M. Guedra, G. Penelet, P. Lotton, and J.-P. Dalmont, "Theoretical prediction of the onset of thermoacoustic instability from the experimental transfer matrix of a thermoacoustic core," $J$. Acoust. Soc. Am., vol. 130, p. 145, 2011.

[11] F. C. Bannwart, G. Penelet, P. Lotton, and J.-P. Dalmont, "Measurements of the impedance matrix of a thermoacoustic core: Applications to the design of thermoacoustic engines," $J$. Acoust. Soc. Am., vol. 133, p. 2650, 2013.

[12] V. Gusev, S. Job, H. Bailliet, P. Lotton, and M. Bruneau, "Acoustic streaming in annular thermoacoustic prime-movers," J. Acoust. Soc. Am., vol. 108, p. 934, 2000.

[13] H. Bailliet, V. Gusev, R. Raspet, and R. A. Hiller, "Acoustic streaming in closed thermoacoustic devices," J. Acoust. Soc. Am., vol. 110, p. 1808, 2001.

[14] S. Backhaus and G. W. Swift, "A thermoacoustic-Stirling heat engine: Detailed study," J. Acoust. Soc. Am., vol. 107, p. 3148, 2000 .

[15] M. E. H. Tijani and S. Spoelstra, "A high performance thermoacoustic engine," J. Appl. Phys., vol. 110, no. 9, pp. 093519093519, 2011.

[16] D. Marx and P. Blanc-Benon, "Numerical simulation of stackheat exchangers coupling in a thermoacoustic refrigerator," AIAA J., vol. 42, no. 7, pp. 1338-1347, 2004.

[17] R. S. Wakeland and R. M. Keolian, "Measurements of the resistance of parallel-plate heat exchangers to oscillating flow at high amplitudes," J. Acoust. Soc. Am., vol. 115, p. 2071, 2004.

[18] A. Berson and P. Blanc-Benon, "Nonperiodicity of the flow within the gap of a thermoacoustic couple at high amplitudes (L)," J. Acoust. Soc. Am., vol. 122, no. 4, pp. EL122-EL127, 2007. 
[19] Y. A. Ilinskii, B. Lipkens, T. S. Lucas, T. W. Van Doren, and E. A. Zabolotskaya, "Nonlinear standing waves in an acoustical resonator," J. Acoust. Soc. Am., vol. 104, no. 5, pp. 2664-2674, 1998.

[20] D. F. Gaitan and A. A. Atchley, "Finite amplitude standing waves in harmonic and anharmonic tubes," J. Acoust. Soc. Am., vol. 93, no. 5, pp. 2489-2495, 1993.

[21] G. W. Swift, "Analysis and performance of a large thermoacoustic engine," J. Acoust. Soc. Am., vol. 92, no. 3, pp. 1551-1563, 1992.

[22] J. Olson and G. W. Swift, "A loaded thermoacoustic engine," J. Acoust. Soc. Am., vol. 98, no. 5, pp. 2690-2693, 1995.

[23] T. Biwa, T. Takahashi, and T. Yazaki, "Observation of traveling thermoacoustic shock waves (L)," J. Acoust. Soc. Am., vol. 130, no. 6, pp. 3558-3561, 2011.

[24] T. Biwa, K. Sobata, S. Otake, and T. Yazaki, "Observation of thermoacoustic shock waves in a resonance tube (L)," J. Acoust. Soc. Am., vol. 136, no. 3, pp. 965-968, 2014.

[25] N. Sugimoto, Nonlinear Waves Motion, ch. Generalized Burgers equations and fractional calculus, pp. 162-179. Longman Scientific \& Technical, 1989.

[26] L. Menguy and J. Gilbert, "Weakly nonlinear gas oscillations in air-filled tubes; solutions and experiments," Acta Acust. united Ac., vol. 86, no. 5, pp. 798-810, 2000.

[27] A. H. Nayfeh and D. T. Mook, Nonlinear oscillations. Wiley, 1979.

[28] M. F. Hamilton, D. T. Blackstock, et al., Nonlinear acoustics, vol. 237. Academic press San Diego, 1998.

[29] W. Chester, "Resonant oscillations in closed tubes," J. Fluid Mech., vol. 18, no. 01, pp. 44-64, 1964.

[30] N. Sugimoto, "Burgers equation with a fractional derivative; hereditary effects on nonlinear acoustic waves," J. Fluid Mech., vol. 225, pp. 631-653, 1991.

[31] W. Chester, "Resonant oscillations of a gas in an open-ended tube," Proc. R. Soc. A, vol. 377, no. 1771, pp. 449-467, 1981.

[32] A. Hirschberg, J. Gilbert, R. Msallam, and A. P. J. Wijnands, "Shock waves in trombones," J. Acoust. Soc. Am., vol. 99, no. 3, pp. 1754-1758, 1996.

[33] J. Gilbert, L. Menguy, and M. Campbell, "A simulation tool for brassiness studies," J. Acoust. Soc. Am., vol. 123, no. 4, pp. 1854-1857, 2008.

[34] P. Merkli and H. Thomann, "Transition to turbulence in oscillating pipe flow," J. Fluid Mech., vol. 68, no. 03, pp. 567-576, 1975.

[35] O. Rudenko and S. I. Soluian, Theoretical foundations of nonlinear acoustics. Consultants Bureau New York/London, 1977.

[36] G. Penelet, S. Job, V. Gusev, P. Lotton, and M. Bruneau, "Dependence of sound amplification on temperature distribution in annular thermoacoustic engines," Acta Acust. united Ac., vol. 91, no. 3, pp. 567-577, 2005.

[37] G. Penelet, V. Gusev, P. Lotton, and M. Bruneau, "Experimental and theoretical study of processes leading to steadystate sound in annular thermoacoustic engines," Phys. Rev. E, vol. 72 , no. 1, p. $016625,2005$.

[38] I. Idelchik, Handbook of Hydraulic Resistance. Begell House Publishers, 2001.

[39] M. Bednarik and P. Konicek, "Propagation of quasiplane nonlinear waves in tubes and the approximate solutions of the generalized burgers equation," J. Acoust. Soc. Am., vol. 112, no. 1, pp. 91-98, 2002 .

[40] P. H. Ceperley, "Gain and efficiency of a short traveling wave heat engine," J. Acoust. Soc. Am., vol. 77, no. 3, pp. 1239-1244, 1985.

[41] H. Hyodo and N. Sugimoto, "Stability analysis for the onset of thermoacoustic oscillations in a gas-filled looped tube," $J$. Fluid Mech., vol. 741, pp. 585-618, 2014.

[42] M. Guedra and G. Penelet, "On the use of a complex frequency for the description of thermoacoustic engines," Acta Acust. united Ac., vol. 98, no. 2, pp. 232-241, 2012. 